neurological deficits during tumor resection in eloquent brain regions. It has been linked with more radical resections to prolong survival in patients harboring brain tumors. We share the experience of awake-craniotomy at our center over the past 5 years.

Materials and Methods: It was a retrospective study from 2014 to 2018. We standardized the procedure (for patient selection, communication, theater preparation) and analyzed the demographics, tumor site and clinical symptomatology of the selected patients, anesthetic agents used, and intraoperative and postoperative complications. Results were analyzed using SPSS software.

Results: A total of 30 patients underwent awakecraniotomy during this period. Majority of the patients were males ( $n=25)$, literate $(n=24)$ belonging to middle socioeconomic status $(n=22)$. The mean age of patients was $38 \pm 11.6$ years with an average weight of $61 \mathrm{~kg}$. Frontal lobe tumors $(n=16)$ were most commonly encountered without any side predilection. Patients mostly presented with convulsions $(n=17)$. Average duration of anesthesia and surgery were 5.8 hours and 4.8 hours, respectively, with a mean awakening duration of $37 \pm 7.7$ minutes. Dexmedetomidine plus fentanyl ( $n=24$ ) was the preferred anesthetic agent along with scalp block. Incidence of intraoperative complications were low; however, postoperative transient neurological deficit was seen in $26.7 \%$ of patients $(n=8)$.

Conclusions: Anesthesia for awake-craniotomy is challenging and anesthesiologists play a pivotal role. With careful patient selection it is well tolerated by them. Planning, coordination, and teamwork are essential for success.

\section{A0033 Use of Dexmedetomidine in Anesthetic Management of a Pregnant Patient with Posterior Fossa Tumor Posted for Ventriculoperitoneal Shunt Roshan Andleeb, ${ }^{1}$ Priyanka Gupta ${ }^{1}$ \\ ${ }^{1}$ Department of Anaesthesiology, All India Institute of Medical Sciences, Rishikesh, Uttarakhand, India}

Background: Neurosurgery during pregnancy is considered a challenge for anesthesiologists due to fetomaternal effects of anesthetic agents. TIVA with adjunct like dexmedetomidine provides optimal condition for neurosurgery, decreases anesthetic requirement, and provides smooth recovery when used in nonpregnant patients although there is limited literature on its use in obstetric patients for neurosurgery.

Case Description: A 28-year-old multigravida with posterior fossa tumor was posted for immediate ventriculoperitoneal shunting at 27th week of gestation. CNS examination revealed no deficit except for drunken gait and past pointing. USG showed 29 weeks active single live fetus. Patient was shifted to OR and standard monitors were attached. Pre-induction patient's vitals and fetal activity were normal. Tococardiography revealed fetal heart rate of 120 breaths/min. Before induction, loading dose of dexmedetomidine $(1 \mu \mathrm{g} / \mathrm{kg}) 50 \mu \mathrm{g}$ followed by maintenance dose at $25 \mu \mathrm{g} / \mathrm{h}(0.5 \mu \mathrm{g} / \mathrm{kg} / \mathrm{h})$ was started. After preoxygenation, RSI was performed by giving inj. fentanyl $100 \mu \mathrm{g}$, inj. propofol
$70 \mathrm{mg}$, and inj. rocuronium $60 \mathrm{mg}$, and intubation was done. Anesthesia was maintained with 50\% oxygen-air mixture, propofol infusion at $27 \mu \mathrm{g} / \mathrm{kg} / \mathrm{min}$, and inj. vecuronium $1 \mathrm{mg}$ bolus as required.

During surgery, maternal hemodynamics were stable. Continuous intraoperative monitoring of fetal activity was done using Doppler. Fetal heart rate varied between 114 and 125 beats/min. Twenty minutes before the completion of surgery, all infusions were stopped and patient was extubated. In postoperative period, fetal activity was confirmed with USG by the obstetrician.

Conclusions: Our case report shows that dexmedetomidine can be used as an adjunct to TIVA for optimal operative condition in neurosurgical procedures with smooth recovery in parturient without any adverse effect on fetomaternal hemodynamics.

\section{A0034 Chin Necrosis during Prone Positioning as a Consequence of Transcranial Motor Evoked Potential Monitoring in Spine Surgery: A Case Report \\ Sunita Doley, ${ }^{1}$ Priyanka Gupta, ${ }^{1}$ Amiya K. Barik ${ }^{1}$ \\ ${ }^{1}$ Department of Anaesthesiology, All India Institute of Medical \\ Sciences, Rishikesh, Uttarakhand, India}

Background: Prone position is commonly used for surgeries of spine, posterior cranial fossa, retroperitoneal structures, gluteal region, and lower limbs. Incidence of iatrogenic neurological injuries is 23.8 to $65.4 \%$ for intramedullary spinal cord tumor resection without evoked potential monitoring. Transcranial motor evoked potential (TcMEP) monitoring is an important tool for intraoperative neurophysiological monitoring of corticospinal tract function during surgery.

Case Description: After taking written informed consent, a case of 40-year-old male, ASA I patient with D5-D7 intramedullary spinal cord tumor was posted for D5-D7 laminectomy and tumor excision. General anesthesia (GA) was induced, and after prone positioning was done with body supported on gel bolsters and head-on-head rest. Intraoperative TCMEP and SSEP monitoring was done, anesthesia was maintained with TIVA of propofol (100-200 $\mu \mathrm{g} / \mathrm{kg} / \mathrm{min})$ and intermittent IV fentanyl. No neuromuscular blocker was used except during intubation. After completion of surgery, patient was turned supine. Redness, swelling, and paresthesia were found over the left side of the chin. All other neurological examinations were within normal limits. On postoperative day 1 , the patch became black and necrotic. Intravenous dexamethasone $4 \mathrm{mg}$ every 8 hours and oral chymotrypsin were given, and hydroheal ointment was applied locally. The patient was followed up, necrosis healed with scar formation after 2 months.

Conclusions: Prolonged surgeries in prone position need meticulous planning of patient positioning. Intermittent careful survey of positioning is important to prevent pressure-related complications, especially when TcMEP monitoring is used. Maintenance of proper hemodynamics, metabolic status, and good coordination with surgeons help avoid position-related complications. 